\title{
PHOTOLUMINESCENCE ENHANCEMENT BY EXCIMER LASER IRRADIATION IN SILICON OXIDE FILMS PREPARED BY PULSED LASER ABLATION
}

\section{Akiharu Morimoto*, Hidetoshi Takizawa, Yasuto Yonezawa ${ }^{+}$, Minoru Kumeda, Tatsuo Shimizu}

Dept. of Electrical and Computer Engineering, Kanazawa University, Kanazawa 920, Japan

Silicon oxide films were prepared at room temperature by pulsed laser ablation using an ArF or $\mathrm{KrF}$ excimer laser in a gas mixture of $\mathrm{He}$ and $\mathrm{O}_{2}$. The effect of an $\mathrm{ArF}$ excimer laser irradiation on the deposited film was investigated. As-deposited transparent films containing Si crystallites with sizes greater than 10-nm show a very weak photoluminescence. However, after laser irradiation with 1000 shots the photoluminescence (PL) intensity was increased by two orders of magnitude. The PL spectrum is centered around $570 \mathrm{~nm}(2.2 \mathrm{eV})$. The origin of the large PL enhancement is discussed.

Keywords: Si oxide, pulsed laser ablation, photoluminescence, laser irradiation

\footnotetext{
* Corresponding author: Akiharu Morimoto,

Dept. of Electrical and Computer Engineering, Kanazawa University, Kodatsuno, Kanazawa 920-8667, JAPAN FAX: +81-76-234-4900, e-mail: amorimot@ec.t.kanazawa-u.ac.jp
}

\footnotetext{
${ }^{+}$On leave from the Industrial Research Institute of Ishikawa, Tomizu, Kanazawa 920-0223, Japan
} 


\section{INTRODUCTION}

The study on luminescent crystalline $\mathrm{Si}$ is an attractive theme of Si physics. Furukawa and Miyasato reported that the visible photoluminescence (PL) from Si oxide films is explained by the quantum-size effect[1]. A variety of silicon-based films containing nanosized crystallites have been prepared for visible PL using various techniques[2,3]. Several models have been presented for the PL mechanism: the quantum confinement effect[4], the effect of radiative defect centers in the $\mathrm{SiO}_{2}$ matrix[5], etc.

Pulsed laser ablation (PLA) has been attracting much attention for the preparation of high-quality perovskite oxide films[6]. This preparation technique has a quite unique feature that the depositing particles have a broad distribution in size from mono- or diatomic species to huge micron-size clusters called droplets. Based on this idea, the preparation of nanosized $\mathrm{Si}$ in various ways and their PL properties have been studied[3, 7]. Si oxide films with nanosized Si crystallites were often annealed in an oxygen environment after the PLA deposition to obtain visible PL. In situ oxidation is attractive for clarifying the oxidation mechanism in the PLA process and for obtaining more efficient PL. So far, the present authors have shown that the He ambient gas promotes the oxidation of the $\mathrm{Si}$ films[8,9]. In this study, the PLA deposition method was employed using a gas mixture of $\mathrm{He}$ and $\mathrm{O}_{2}$ for the preparation of inhomogeneous oxide film with Si crystallites in Si oxide matrix. The films were then irradiated by the pulsed laser to obtain visible PL.

\section{EXPERIMENTAL}

Silicon oxide films about $1.5 \mu \mathrm{m}$-thick were prepared on fused quartz substrates at room temperature by PLA using a Si target. The excimer laser employed was mainly an ArF excimer laser with a wavelength of $193 \mathrm{~nm}$, a pulsed duration of $10 \mathrm{~ns}$ and a repetition rate of $5 \mathrm{~Hz}$. The ambient gas was a mixture of $130 \mathrm{~Pa}$ of $\mathrm{He}$ and of $1.1 \mathrm{~Pa}$ of $\mathrm{O}_{2}$. The laser beam was focused to a 
small area of $1 \mathrm{~mm} \times 2 \mathrm{~mm}$, resulting in a fluence of $3.5 \mathrm{~J} / \mathrm{cm}^{2}$. Films prepared by the $\mathrm{KrF}$ excimer laser with a wavelength of $248 \mathrm{~nm}$ were also used, for comparison. The as-deposited films show a weak PL. To enhance the PL without thermal annealing we performed an ArF excimer laser irradiation with a fluence of $0.5 \mathrm{~J} / \mathrm{cm}^{2}$ and a repetition rate of $1 \mathrm{~Hz}$ on the deposited films. ArF excimer laser irradiation with a repetition rate of $5 \mathrm{~Hz}$ was also performed, for comparison.

X-ray diffraction (XRD), optical absorption, PL, Raman scattering, and electron spin resonance (ESR) measurements were performed at room temperature. The PL and Raman scattering measurements were carried out using a JOBIN YVON double monochrometer of with Ar-laser excitation $(488 \mathrm{~nm})$. To obtain the spectral response, a calibration of the detection system was performed using a standard halogen lamp.

\section{RESULTS}

There is no essential difference in the properties of the as-deposited films prepared using ArF and $\mathrm{KrF}$ excimer lasers. The XPS Si $2 p$ spectrum has a peak around a binding energy of $103.7 \mathrm{eV}$ with a small tail toward the low binding-energy side, resulting in a quite small component around $99 \mathrm{eV}$. This result suggests that the as-deposited films were almost stoichiometric $\mathrm{SiO}_{2}$ films with a quite small fraction of $\mathrm{Si}$ clusters. As shown later the Raman spectrum for the as-deposited film shows a narrow component located near $520 \mathrm{~cm}^{-1}$, suggesting the formation of $\mathrm{Si}$ crystallites of size greater than $10 \mathrm{~nm}$ based on the results of previous reports[10, 11]. This finding is consistent with the result of the XRD measurement, which shows diffraction peaks originating from Si crystallites with a size above $10 \mathrm{~nm}$.

Figure 1 shows the changes in the PL spectrum with pulsed-laser-irradiation up to 3000 shots. The as-deposited film, which was prepared using ArF excimer laser, was irradiated by the ArF excimer laser with a repetition rate of $1 \mathrm{~Hz}$. The ArF excimer laser irradiation onto the 
as-deposited film is found to cause a dramatic increase in the PL intensity with a green-red visible spectrum (centered around $570 \mathrm{~nm}$ or $2.2 \mathrm{eV}$ ) while the as-deposited film shows only a weak PL. Excess shots of the laser irradiation, however, caused a serious decrease in the PL intensity. We should pay attention to the possible role of substrate because fused quartz substrate is well known to show a strong PL in this wavelength range after an intense UV laser irradiation. To ascertain that the enhanced PL is really produced by the Si oxide film, the same ArF excimer laser irradiation was performed on the fused quartz substrate alone. This experiment revealed that the PL intensity with the level of the as-deposited film in Fig. 1 was remained unchanged by the excimer laser irradiation with the present fluences and the numbers of shots.

Figure 2 shows the PL peak intensity (a) and the PL peak wavelength (b) as a function of the number of shots. The data were derived from Fig. 1. Figure 2(a) shows that the PL peak intensity increases by two orders of magnitude and Fig. 2(b) shows that the PL peak wavelength changes from $550 \mathrm{~nm}$ to $570 \mathrm{~nm}$ with an increase in the number of shots. Depending on the laser used in the PLA and the repetition rate of the irradiation laser, the PL intensity and peak wavelength behavior versus the number of shots is quantitatively different. Qualitatively speaking, however, there is no essential difference in the PL behavior for the present preparation and irradiation conditions.

To analyze the film structure, Raman scattering measurement was performed. Figure 3 shows the change in the Raman spectrum with irradiation by pulsed laser up to 3000 shots on the deposited film. The film deposited using the ArF excimer laser was irradiated by the ArF excimer laser at a repetition rate of $1 \mathrm{~Hz}$. The Raman scattering measurement show that a peak originating from the Si crystallites around $520 \mathrm{~cm}^{-1}$ for the as-deposited and irradiated films, suggesting that Si crystallites larger than $10 \mathrm{~nm}$ are formed. No broad band around $480 \mathrm{~cm}^{-1}$ which would indicate the presence of amorphous Si was detected in a wide range scan. These findings suggest that Si crystallites rather than amorphous Si clusters are present in these films. 
A wide scan over $1000 \mathrm{~cm}^{-1}$ was not performed for the detection of $\mathrm{SiO}_{\mathrm{x}}$ because the signal should be obscured by the signal from the substrate.

Figure 4 shows the peak wavenumber (a) and the full width at half maximum (FWHM) (b) of the Raman spectrum as a function of the number of shots. The data were derived from Fig. 3. The peak wavenumber does not change much with an increase in the number of shots. Although there is some scattering in the data, the peak wavenumber appears to be slightly decreased with an increase in the number of shots. The FWHM of the Raman scattering spectra does not change appreciably with an increase in the number of shots.

\section{DISCUSSION}

It is difficult to estimate accurately our Si-crystallite size because the XPS, the Raman and XRD measurements themselves do not tell us an accurate crystallite size. Based on the results of these measurements the Si-crystallite size is approximately estimated to be larger than $10 \mathrm{~nm}$ while the volume fraction of the crystallites to the amorphous oxide appears to be much below $10 \%$.

The He gas pressure for the preparation of Si oxide films was $130 \mathrm{~Pa}$. Using scanning electron microscopy (SEM) Yoshida et al. reported that the mean diameter of Si crystallites prepared in pure He ambient gas was determined by the ambient pressure[12]. Based on their result, the mean diameter of the Si crystallites for the present films, which were prepared in a gas mixture of $\mathrm{He}$ and $\mathrm{O}_{2}$, was estimated to be about $10 \mathrm{~nm}$. The final diameter of the Si crystallites after oxidation by the ambient $\mathrm{O}_{2}$ in the present PLA process is expected to be smaller than 10 $\mathrm{nm}$. The size of the Si crystallites in the present study, however, is above $10 \mathrm{~nm}$ as described above. This difference is possibly originating from a difference in the oxidation process and differences in the target-substrate spacing and the laser fluence.

Both quantum confinement in $\mathrm{Si}$ crystallites and defect luminescence in the $\mathrm{SiO}_{\mathrm{x}}$ matrix 
are possible mechanisms for the 2.2-eV-PL observed after the ArF-excimer-laser irradiation.

Let us discuss the first mechanism. Takigawa et al. and Ohmukai et al. reported that Ar excimer-laser irradiation onto exactly stoichiometric $\mathrm{SiO}_{2}$ and $\mathrm{Si}_{3} \mathrm{~N}_{4}$ causes the precipitation of Si crystallites in the surface layer, respectively[13,14]. They ascribed the precipitation to photochemical breaking of $\mathrm{Si}-\mathrm{O}$ or $\mathrm{Si}-\mathrm{N}$ bonds, and desorption of $\mathrm{O}$ or $\mathrm{N}$ atoms. In their cases, the energy of the excitation laser is just above the band gap of the materials. The present Si oxide films has a finite absorption coefficient at the wavelength of the ArF excimer laser (193 nm) because the $\mathrm{O}$ content is slightly below the stoichiometric one. Therefore, the precipitation and crystallization of $\mathrm{Si}$ induced by the ArF-excimer-laser irradiation should be possible through bond breaking process caused by electronic excitations from the valence band to the conduction band and/or a successive temperature increase. The slight shift of the Raman peak toward the lower wavenumber does not contradict the formation of new small Si crystallites in addition to the large Si crystallites of the as-deposited film. The FWHM is almost unchanged because the resolution of the Raman measurement is around $5 \mathrm{~cm}^{-1}$. The Si $2 \mathrm{p}$ XPS spectrum was also slightly extended to the low binding energy side by the laser irradiation, supporting the possibility of the precipitation of Si atoms.

Next, let us discuss the second mechanism. In the $2 \mathrm{eV}$ to $3 \mathrm{eV}$ range, the PL spectrum could be ascribed to various defects; e. g., the nonbridging oxygen hole center (NBOHC) mainly stabilized by $\mathrm{H}[15]$, or the Si-excess defects [16], the defect related to $\mathrm{OH}$ adsobate[17] in porous Si or various Si oxides. Since the present oxide films were prepared by PLA without any intentionally incorporation of $\mathrm{H}$, it offers improbable that the H-related defects is responsible for the PL. To clarify the origin of the PL enhancement, an ESR measurement was performed on the as-deposited and laser-irradiated films. The ESR signal consists of two components: a narrow component around $\mathrm{g}=2.000$ and a broad component around $\mathrm{g}=2.005$. The former corresponds to Si dangling bonds backbonded by three $\mathrm{O}$ atoms (the $\mathrm{E}^{\prime}$ center), and the latter to Si dangling 
bonds backbonded by three $\mathrm{Si}$ atoms[18]. The defect density with $\mathrm{g}=2.005$ is estimated to be about $1 \times 10^{17} \mathrm{~cm}^{-3}$ for the as-deposited film and it does not increase with laser irradiation. This suggests that the defect with $\mathrm{g}=2.005$ is not the origin of the PL induced by the laser irradiation because the large PL enhancement is not consistent with the nearly constant defect density. Since the substrates used are fused quartz, it is difficult to estimate the density of $E^{\prime}$ centers in the Si-oxide films. Thus there remains a possibility that the PL enhancement is due to an increase in the E'-center density.

\section{CONCLUSIONS}

As-deposited transparent films containing Si crystallites with a size larger than $10 \mathrm{~nm}$ show a very weak PL. However, after laser irradiation of 1000 shots the PL intensity was increased by two orders of magnitude. The PL spectrum was centered around $570 \mathrm{~nm}(2.2 \mathrm{eV})$. The possible mechanisms were discussed, related to quantum confinement effect or defect luminescence.

\section{ACKNOWLEDGEMENTS}

We thank T. Sasaga and K. Nagai of Kanazawa Univ. for technical assistance. We are grateful to Shibuya Kogyo Co., Ltd. for supplying the ArF excimer laser system. This work was

partly supported by Grant-in-Aid for Scientific Research (C) No. 07650008 from the Ministry of Education, Science, Sports and Culture of Japan.

\section{REFERENCES}

[1] S. Furukawa, T. Miyasato, Jpn. J. Appl. Phys. 27 (1988) L2207.

[2] L. T. Canham, Appl. Phys. Lett. 57 (1990) 1046.

[3] E. Werwa, A. A. Seraphin, L. A. Chiu, C. Zhou, K. D. Kolenbrander, Appl. Phys. Lett. 64 
(1994) 1821.

[4] Y. Yamada, T. Orii, I. Umezu, S. Takeyama, T. Yoshida, Jpn. J. Appl. Phys. 35 (1996) 1361.

[5] K. S. Min, K. V. Shcheglov, C. M. Yang, H. A. Atwater, Appl. Phys. Lett. 68 (1996) 2511.

[6] D. Dijkkamp, T. Venkatesan, X. D. Wu, S. A. Shaheen, N. Jisrawi, Y. H. Min-Lee, W. L. McLean, and M. Croft, Appl. Phys. Lett. 51 (1987) 619.

[7] T. Makimura, Y. Kunii, K. Murakami, Jpn. J. Appl. Phys. 35 (1996) 4780.

[8] A. Masuda, K. Matsuda, S. Usui, Y. Yonezawa, T. Minamikawa, A. Morimoto, T. Shimizu, Mater. Sci. \& Eng. B41 (1996) 161.

[9] A. Masuda, S. Usui, Y. Yamanaka, Y. Yonezawa, T. Minamikawa, A. Morimoto, M. Kumeda, T. Shimizu, (submitted to Jpn. J. Appl. Phys.)

[10] H. Richter, Z. P. Wang, and L. Ley: Solid State Commun. 39 (1981) 625.

[11] I. H. Campbell, and P.M. Fauchet: Solid State Commun. 58 (1986) 739.

[12] T. Yoshida, S. Takeyama, Y. Yamada, K. Mutoh, Appl. Phys. Lett. 68 (1996) 1772.

[13] Y. Takigawa, K. Kurosawa, W. Sasaki, K. Yoshida, E. Fujiwara, Y. Kato, J. Non-Cryst. Sol. 116 (1990) 293.

[14] M. Ohmukai, H. Naito, M. Okuda, K. Kurosawa, W. Sasaki, T. Matsushita, Y. Tsunawaki, S. Nozawa, T. Igarashi, Jpn. J. Appl. Phys. 32 (1993) L1062.

[15] S. M. Prokes, O. J. Glembocki, Phys. Rev. B49 (1994) 2238.

[16] T. Shimizu-Iwayama, Y. Terao, A. Kamiya, M. Takeda, S. Nakao, K. Saitoh, Thin Solid Films, 276 (1996) 104.

[17] H. Tamura, M. Ruckschloss, T. Wirschem, S. Veprek, Appl. Phys. Lett. 65 (1994) 1537.

[18] A. Morimoto, H. Noriyama, T. Shimizu, Jpn. J. Appl. Phys. 26 (1987) 22. 


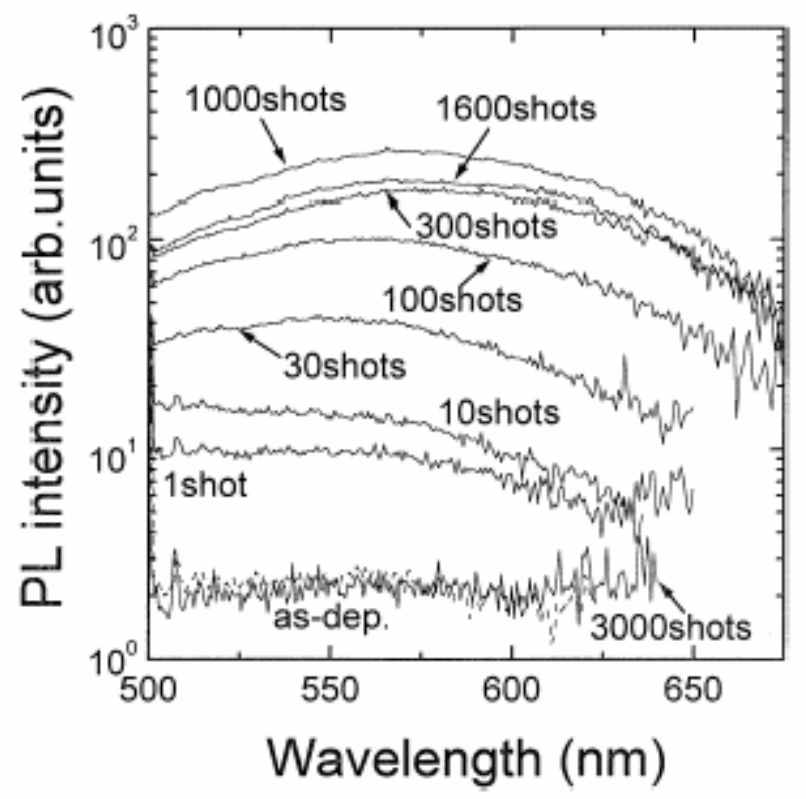

Fig. 1. Changes in the PL spectrum with pulsed laser irradiation, up to 3000 shots. The film deposited using an ArF excimer laser was then irradiated by ArF excimer laser with a repetition rate of $1 \mathrm{~Hz}$.

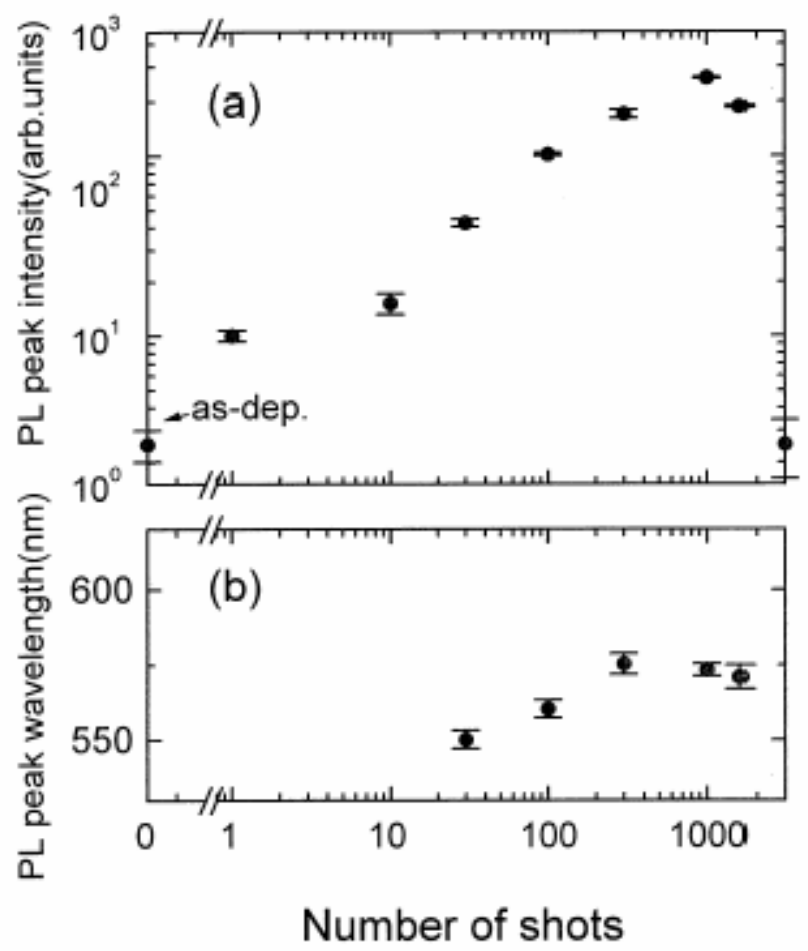

Fig. 2. Peak intensity (a) and peak wavelength (b) of the PL spectrum as a function of the number of shots. The data were derived from Fig. 1. 


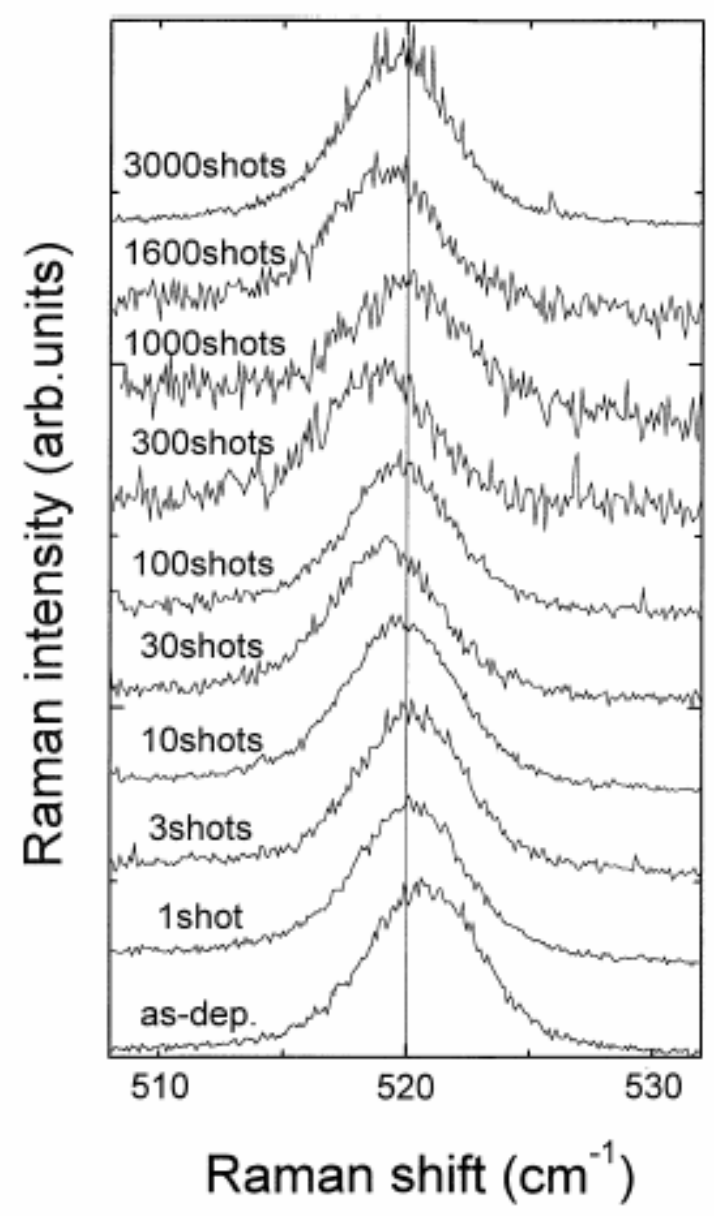

Fig. 3. Raman spectra for films irradiated by pulsed laser up to 3000 shots. The film deposited using an ArF excimer laser was then irradiated by an $\mathrm{ArF}$ excimer laser with a repetition rate of $1 \mathrm{~Hz}$. 


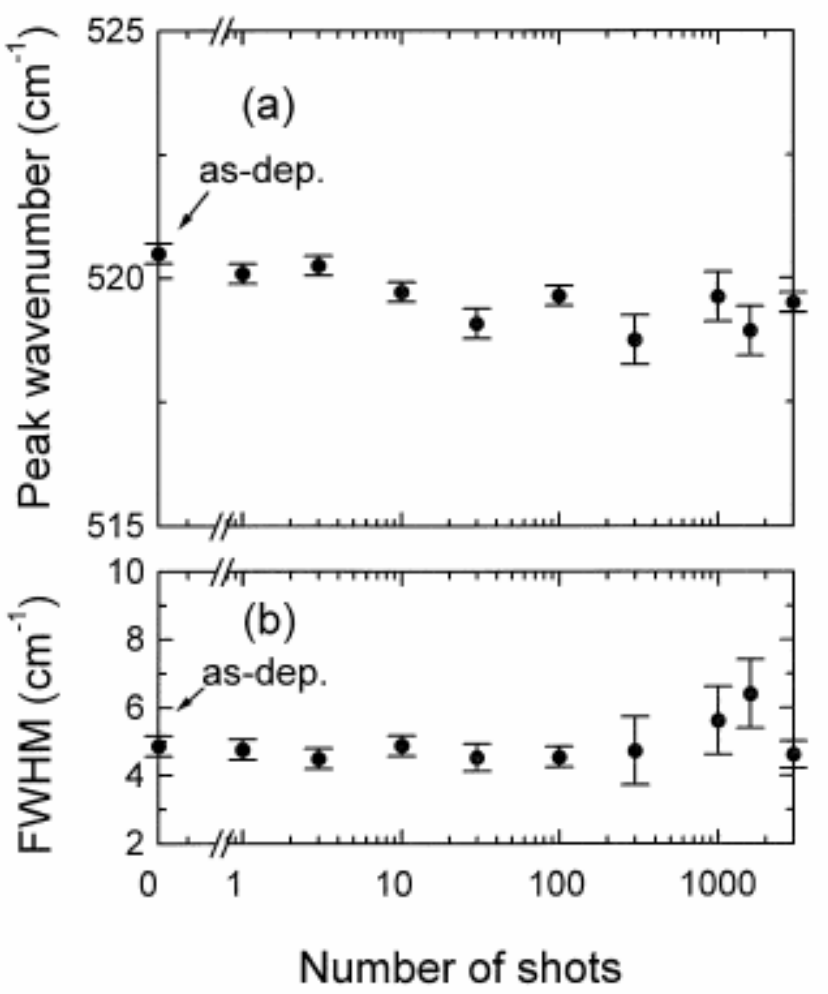

Fig. 4. Peak wavenumber (a) and full width at half maximum (FWHM) (b) of the Raman scattering spectra as a function of the number of shots. The data were derived from Fig. 3.

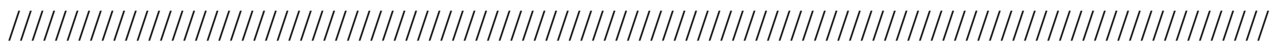

2543 words $+150 \times 4$ figures $=3143$ words $\quad$ limit: 3,200 words 